\title{
Geburtsstunde des modernen Steuerstaats in Deutschland 1919/1920
}

\author{
Vor 100 Jahren haben die „Erzbergerschen Steuer- und Finanzreformen“ die öffentlichen \\ Finanzen in Deutschland grundlegend umgestaltet. Sie zentralisierten die Finanzverfassung \\ und modernisierten das Steuersystem umfassend. Einkommensteuer, Körperschaftsteuer und \\ Erbschaftsteuer wurden deutschlandweit vereinheitlicht und deutlich erhöht, ferner wurden eine \\ Vermögensabgabe eingeführt und die indirekten Steuern ausgebaut. Die gesamtwirtschaftliche \\ Steuer- und Abgabenbelastung verdoppelte sich bis 1925. Diese Reformen prägen die \\ Grundlagen der öffentlichen Finanzen und des Steuersystems bis heute.
}

Vor 100 Jahren entstanden in Deutschland die wesentlichen Grundlagen des heutigen Steuersystems. In einem beispiellosen Kraftakt wurden von Juli 1919 bis März 1920 umfassende Steuer- und Finanzreformen durchgesetzt maßgeblich vorangetrieben vom damaligen Reichsfinanzminister Matthias Erzberger, dem führenden Politiker des linken Flügels der Zentrumspartei. ${ }^{1}$

\begin{abstract}
Hintergrund war die desaströse Lage der öffentlichen Finanzen nach der militärischen Niederlage im Ersten Weltkrieg. Trotz (oder wegen) des extremen Zeitdrucks, der schwierigen Wirtschaftslage und des politischen Umbruchs gelang eine nahezu vollständige Umgestaltung, Modernisierung und Zentralisierung der Besteuerung und
\end{abstract}

(c) Der/die Autor(en) 2020. Open Access: Dieser Artikel wird unter der Creative Commons Namensnennung 4.0 International Lizenz (https:// creativecommons.org/licenses/by/4.0/deed.de) veröffentlicht.

Open Access wird durch die ZBW - Leibniz-Informationszentrum Wirtschaft gefördert.

1 K. Epstein: Matthias Erzberger und das Dilemma der deutschen Demokratie, Frankfurt a. M. 1976, S. $373 \mathrm{ff}$

Dr. Stefan Bach ist wissenschaftlicher Mitarbeiter am Deutschen Institut für Wirtschaftsforschung (DIW) in Berlin.

Dr. phil. Marc Buggeln ist Visiting Fellow in Clare Hall (Cambridge) und Gastdozent am Historischen Institut der Universität Cambridge. der öffentlichen Finanzen. ${ }^{2}$ Deren wesentliche Elemente haben bis heute Bestand: die Grundstrukturen des Steuersystems und der Finanzverwaltung, die Abgabenordnung sowie der zentralistische kooperative Finanzföderalismus. Auch bei Aufkommen und Belastungen markieren diese Reformen den Durchbruch des modernen Steuerund Wohlfahrtsstaats in Deutschland. Das Steueraufkommen verdoppelte sich nahezu bis 1925. Ohne Übertreibung kann man von einer Jahrhundertreform sprechen - die einzige wirklich grundlegende Steuer- und Finanzreform, die es in Deutschland seit dem 19. Jahrhundert bis heute gegeben hat.

„Der Krieg ist der Verwüster der Finanzen“ - mit diesen Worten eröffnete Erzberger seine erste Rede als Reichsfinanzminister vor der Weimarer Nationalversammlung am 8. Juli 1919. ${ }^{3}$ Finanzpolitisch war das Deutsche Reich schlecht gerüstet in die Exzesse des Ersten Weltkriegs gegangen. In den Jahren vor 1914 lag die gesamtwirtschaftliche Steuerquote bei etwa $8 \%$ des Bruttoinlandsprodukts (BIP). ${ }^{4}$ Hinzu kamen Sozialbeiträge von $2 \%$ des BIP sowie Erwerbseinkommen von bis zu $2 \%$ des BIP, vor allem aus den Überschüssen der Staatseisenbahnen.

Die Gesetzgebungskompetenzen zur Besteuerung und die Steuereinnahmen lagen weitgehend bei den Bundesländern. Diese hatten begonnen, ihre Steuersysteme zu

2 Eine zeitgenössische Übersicht gibt R. van der Borght: Die Reichssteuergesetze von 1919, in: Finanzarchiv, 37. Jg. (1920) H. 1, S. $136-$ 222, ders.: Die Reichssteuergesetze von 1919/20 (Schlussbericht), in: Finanzarchiv, 37. Jg. (1920), H. 2, S. 80-92; H.-P. Ullmann: Der deutsche Steuerstaat. Geschichte der öffentlichen Finanzen vom 18. Jahrhundert bis heute, München 2005, S. $101 \mathrm{ff}$.

3 M. Erzberger: Einführungsrede, gehalten am 8.7.1919 in der Nationalversammlung, in: ders.: Reden zur Neuordnung des deutschen Finanzwesens, Berlin 1919, S. 3.

4 M. Spoerer: Öffentliche Finanzen, in: T. Rahlf (Hrsg.): Deutschland in Daten, Zeitreihen zur Historischen Statistik, Bonn 2015, S. 106. 
modernisieren. Prägend war die Entwicklung in Preußen, das im Zuge der „Miquelschen Steuerreformen" 1891 bis 1893 seine Steuern neu geordnet und eine moderne Einkommensteuer sowie eine ergänzende Vermögensteuer eingeführt hatte. ${ }^{5}$ Aufkommen und Belastungen von höheren Einkommen und Vermögen blieben aber niedrig, unter anderem, weil der Spitzensteuersatz der Einkommensteuer in allen Bundesländern unter $10 \%$ lag. Das Reich war finanziell nur schwach ausgestattet. Seine Einnahmen beruhten vor allem auf Zöllen und Verbrauchsteuern. Darüber hinaus war es auf Zuweisungen der Länder angewiesen (Matrikularbeiträge) und galt daher als „Kostgänger der Länder“. Bereits vor dem Ersten Weltkrieg hatten hohe Rüstungsausgaben den Reichshaushalt belastet und die Schulden deutlich erhöht. Die zumeist stark konservativ geprägte Reichstagsmehrheit und die Bundesländer blockierten die Übertragung der Besteuerung von Einkommen und Vermögen auf das Reich, da man befürchtete, dass das umfassende Wahlrecht auf Reichsebene die Macht der Sozialdemokratie weiter ausweiten und diese dann die Wohlhabenden mit progressiven Steuern schwer belasten würden. In den Bundesländern galt zumeist ein Zensuswahlrecht wie das Dreiklassenwahlrecht in PreuBen, bei dem die Stimmen der wohlhabenden Bevölkerung stärker gewichtet wurden.

Die immensen Kriegskosten des Ersten Weltkriegs wurden weitgehend mit Schulden finanziert. Nachdem sich der Krieg in die Länge zog, enorme Opfer forderte und immer höhere Kosten verschlang, führte man Kriegsgewinnsteuern und eine allgemeine Umsatzsteuer ein. Diese konnten die riesigen Haushaltsdefizite aber nur geringfügig vermindern. Die Verschuldung stieg weiter und wurde zunehmend durch Geld- und Kreditschöpfung dominiert, was die Geldmenge ausweitete und die Inflation anheizte. ${ }^{6}$

Nach der militärischen Niederlage drohte der finanzielle Kollaps. Die gesamte Staatsverschuldung lag Ende 1919 bei über 200 Mrd. Mark ${ }^{7}$ und damit bei mindestens $150 \%$ des BIP. Hinzu kamen hohe laufende Defizite durch den Schuldendienst, Ausgaben für Demobilisierung, Wiederankurbelung der zivilen Wirtschaft, Besatzungskosten und Kriegsopferversorgung. Ferner drohten massive Reparationsforderungen der Sieger.

Bereits seit der Mitte des Krieges entwickelte die kaiserliche Reichsregierung Konzepte für Steuererhöhungen

5 A. Thier: Steuergesetzgebung und Verfassung in der konstitutionellen Monarchie. Steuerreformen in Preußen 1871-1893, Frankfurt a. M. 1999.

6 M. Buggeln: Das Versprechen der Gleichheit. Progressive Steuern und die Reduktion sozialer Ungleichheit 1871-1945, Berlin 2019 (Habilitationsschrift HU Berlin), S. 178-244.

7 M. Erzberger: 4. Rede gehalten am 30. Oktober 1919 in der Nationalversammlung, in: ders.: Reden, a. a. O., S. 82. und eine Zentralisierung der Staatsfinanzen beim Reich. Unmittelbar nach Waffenstillstand und politischem Umbruch im November 1918 begannen die Planungen zu umfassenden Steuer- und Finanzreformen. ${ }^{8}$ Ende Dezember 1918 wurden erste Leitlinien vorgestellt: Einführung einer außerordentlichen Kriegsgewinnsteuer; Belastung der restlichen Vermögen mit einer allgemeinen Vermögensabgabe in Fortführung der bereits vor dem Krieg bestehenden Besitzsteuer; Einführung einer Reichseinkommensteuer; Erschließung neuer Steuerquellen, wie Kapitalertrag- und Betriebsertragsteuer; starke Erhöhung der Erbschaftsteuer; Einführung einer Abgabenordnung, um die Gesetze wirksam durchzusetzen.

Aus den Wahlen zur Nationalversammlung am 19. Januar 1919 ging die (Mehrheits-)SPD mit 37,9\% der Stimmen als Sieger hervor. Zusammen mit der katholischen Zentrumspartei (19,7\%) und der linksliberalen Deutschen Demokratischen Partei (DDP) (18,5\%) bildete sie eine Koalitionsregierung (Weimarer Koalition). Angesichts der anhaltenden bürgerkriegsähnlichen Zustände in Berlin tagte die Nationalversammlung ab Anfang Februar 1919 in Weimar. Nach der Verabschiedung eines Gesetzes über die vorläufige Reichsgewalt wurde der Sozialdemokrat Friedrich Ebert zum Reichspräsidenten gewählt und eine Reichsregierung unter dem Sozialdemokraten Philipp Scheidemann gebildet. Eugen Schiffer und ab April 1919 Bernhard Dernburg (beide DDP) übernahmen das neu geschaffene Reichsfinanzministerium. Auch Matthias Erzberger gehörte der Regierung als Minister ohne Geschäftsbereich an. Er war weiterhin für den Waffenstillstand und die Friedensverhandlungen zuständig, mit denen er bereits von der letzten kaiserlichen Regierung betraut worden war und als Delegationsleiter den Waffenstillstand unterzeichnet hatte. Deswegen wurde er von der extremen Rechten in Deutschland stark angefeindet.

\section{Zentralisierung der Finanz- und Steuerkompetenzen}

Die vorrangige Aufgabe der Nationalversammlung war es, eine neue Verfassung zu beschließen, zugleich übte sie als Parlament die laufende Gesetzgebung und Regierungskontrolle aus. Bei der Neuordnung der öffentlichen Finanzen ging es neben der Modernisierung und Ausweitung vor allem um die Kompetenzverteilung in der neuen bundesstaatlichen Ordnung. Dass dem Reich deutlich größere Finanzkompetenzen zulasten der Länder zukommen mussten, war allen Beteiligten klar. Umstritten war, wie weit der Zugriff auf die bisher weitgehend den Län-

8 M. Hacker: Gibt es „Gerechtigkeit“ in der Steuerpolitik? Der politischphilosophische Diskurs über Recht und Gerechtigkeit am Beispiel der Entstehung des modernen Einkommensteuerrechts in der Weimarer Republik, Berlin 2013 (Dissertation FU Berlin), S. 105 ff. 
dern vorbehaltenen „direkten“ Steuern auf Einkommen und Vermögen gehen sollten. Die Länder hatten dazu starke Vorbehalte. Viele wollten das Reich weiterhin auf die indirekten Steuern beschränken und dazu die Umsatzsteuer als allgemeine Verbrauchsteuer ausbauen.

Angesichts der dramatischen Finanzlage erwies sich das als unrealistisch. Im Mai 1919 stimmten die Länder im Grundsatz einer einheitlichen Einkommensteuer des Reichs zu, an deren Aufkommen die Länder und Gemeinden beteiligt werden sollten. Im Verfassungsausschuss der Nationalversammlung wurde die Gesetzgebungskompetenz des Reichs für grundsätzlich alle Steuern und Abgaben festgelegt, dabei sollte es auf die Lebensfähigkeit der Länder Rücksicht nehmen. ${ }^{9}$ Der Reichsrat als Länderkammer konnte gegen vom Reichstag beschlossene Gesetze ein Veto einlegen, das allerdings mit Zweidrittelmehrheit des Reichstags überstimmt werden konnte (Artikel 74 Weimarer Reichsverfassung - WRV).

Im Juni 1919 zerbrach die Reichsregierung unter Philipp Scheidemann (SPD) im Streit um die Annahme des Versailler Friedensvertrags. Daraufhin wurde am 21. Juni eine neue Regierung mit Gustav Bauer (SPD) als Ministerpräsident gebildet, die sich weiterhin auf die Weimarer Koalition in der Nationalversammlung stützte. Bauer und Ebert ersuchten Erzberger ausdrücklich, das Amt des Reichsfinanzministers zu übernehmen, da sie bei ihm den Willen, die Tatkraft und das politische Geschick erkannten, die umfassenden Steuer- und Finanzreformen zügig durchzusetzen. Um seine Rolle zu unterstreichen, wurde er zugleich zum Vizekanzler berufen.

Nach der Annahme des Friedensvertrags durch Regierung und Nationalversammlung endete Ende Juni 1919 der Kriegszustand, die Seeblockade der Alliierten wurde sukzessive gelockert. Ferner wurden die Beratungen zur Verfassung zügig abgeschlossen. Sie wurde Anfang August 1919 verabschiedet. Damit waren die politischen und verfassungsrechtlichen Voraussetzungen für die Reformen der Steuer- und Finanzordnung gegeben, deren Umsetzung Erzberger und seine Mitstreiter umgehend in Angriff nahmen.

Erzberger und das Reichsfinanzministerium konnten sich grundsätzlich auf die weitgehende Unterstützung der Koalitionsfraktionen in der Nationalversammlung verlassen. Sie stimmten sich insbesondere mit der SPD und ihrem einflussreichen finanzpolitischem Sprecher Wilhelm Keil

9 Artikel 8 der Verfassung des Deutschen Reichs vom 11.8.1919: Weimarer Reichsverfassung (WRV). ab. ${ }^{10}$ Die Sozialdemokraten hatten seit Jahrzehnten eine Zentralisierung der Steuerpolitik beim Reich, eine Stärkung der progressiven Einkommens- und Vermögensbesteuerung sowie deren effektive Durchsetzung gefordert, was nun auf der Agenda stand. Auch die linksliberale DDP war unitaristisch orientiert und mit der Zentralisierung der Finanzverfassung grundsätzlich einverstanden. Sie war allerdings zurückhaltender bei der Ausweitung der Steuerbelastung sowie hinsichtlich des Auf- und Ausbaus der Steuerverwaltung. Erzbergers Partei, das traditionell föderalistische Zentrum, war eher skeptisch, vor allem der konservative Flügel und die Bayerische Volkspartei (BVP), die sich in Bayern vom Zentrum abgespalten hatte, jedoch in der Fraktionsgemeinschaft verblieb. Sie konnten sich aber dem Druck der prekären Finanzlage und der Regierungsverantwortung nicht entziehen und wurden von Erzberger eingebunden. In Verwaltungsapparaten, Ländern, Verbänden und in der breiten Öffentlichkeit überwogen jedoch Zurückhaltung und zunehmender Widerstand angesichts der umfassenden Reformen und der massiven Steuererhöhungen. Daher war es eine beachtenswerte Leistung, das umfangreiche Reformwerk zügig durchzusetzen.

\section{Reichsfinanzverwaltung und Steuerverbund}

Eine zentrale Weichenstellung und politische Meisterleistung zu Beginn des Reformprozesses im Sommer 1919 war die schnelle und konsequente Zentralisierung der Finanz- und Steuerkompetenzen beim Reich, einschließlich der Steuererhebung durch die neu aufzubauende Reichsfinanzverwaltung. ${ }^{11}$ „Der große Steuersouverän der Zukunft kann nur das einige Deutsche Reich sein" erklärte Erzberger vor der Nationalversammlung. ${ }^{12}$ Dies löste naturgemäß anhaltenden Widerspruch bei den Ländern aus, die bereits ihre Steuerkompetenzen weitgehend eingebüßt hatten. Erzberger und die Finanzpolitiker der Koalition erkannten, dass für die effektive und gleichmäßige Erhebung der beträchtlichen Steuererhöhungen eine eigenständige professionelle Finanzverwaltung, die dem Reich unterstellt werden sollte, wesentlich war. In den Ländern gab es zumeist keine Fachverwaltung für Finanzen, Steuern und Abgaben. Diese Aufgaben wurden von der inneren Verwaltung betreut. Entsprechend unzulänglich und ungleichmäßig war die Durchsetzung der Steuerpflicht. Erst einzelne Länder wie Württemberg hatten mit dem Aufbau einer eigenständigen Fachverwaltung für Steuern und Finanzen begonnen.

10 A. Möller: Reichsfinanzminister Matthias Erzberger und sein Reformwerk, Bonn 1971, S. 17 ff., S. 29 ff.

11 A. Schmidt-Essen: Reichseinkommensteuer und Einheitsstaat, in: Wirtschaftsdienst, H. 29 vom 18.7.1919; A. Möller, a.a. O., S. 29 ff.

12 M. Erzberger, Eröffnungsrede, a.a.O., S. 7. 
Der Gesetzentwurf zur Abgabenordnung einschließlich der Regelungen zur Reichsfinanzverwaltung wurde unter großem Zeitdruck fertiggestellt und in die Nationalversammlung eingebracht. ${ }^{13}$ Durch zähe Verhandlungen mit den Ländern erreichten Erzberger und seine Mitstreiter die Zuständigkeit der Reichsfinanzverwaltung für fast alle Steuern. Baden, Bayern und Sachsen, die sich bis zuletzt gesträubt hatten, wurden über Zugeständnisse eingebunden. So konnten die Länder bei der Einrichtung der Landesfinanzämter als Mittelbehörden mitwirken. Erzberger stellte den reformkritischen Landesfinanzministern eine Ernennung zu Landesfinanzamtspräsidenten auf Lebenszeit in Aussicht, sollten sie der Reform zustimmen. ${ }^{14}$ Der erste Teil zur Organisation der Finanzbehörden im Entwurf der Abgabenordnung wurde dann als gesondertes "Gesetz über die Reichsfinanzverwaltung“ eingebracht und noch vor der Sommerpause verabschiedet, damit der Aufbau der Reichsfinanzverwaltung umgehend im Herbst beginnen konnte. Die Regelungen wurden im Dezember 1919 wieder ohne wesentliche Änderungen als erster Teil in die insgesamt angenommene Reichsabgabenordnung integriert.

Auf dieser Grundlage wurde dann eine spezialisierte und professionalisierte Finanzverwaltung aufgebaut. Ferner entstand ein eigenständiges Steuerverwaltungsrecht, das im Wesentlichen bis heute gilt. Mit dem Reichsfinanzhof existierte bereits eine oberste Spruchkammer für die Umsatzsteuer und die Verbrauchsteuern, die in den folgenden Jahren als eigenständige Finanzgerichtsbarkeit für alle Steuern mit Landesfinanzgerichten als regionalem Unterbau ausgebaut wurde. Auch die Steuerberatung entwickelte sich in den Folgejahren zu einem eigenständigen freien Beruf, was vor allem an der massiven Erhöhung der Steuersätze lag, die bei Unternehmen und wohlhabenden Bürgern einen Beratungsbedarf entstehen ließen.

Nach weiteren zähen Verhandlungen wurde im März 1920 das Landessteuergesetz verabschiedet, das den neuen Finanzausgleich zwischen Reich, Ländern und Gemeinden regelte..$^{15}$ Das bisher geltende Trennsystem der Steuerquellen wurde durch ein Verbundsystem abgelöst, bei dem die Gebietskörperschaften mit Anteilen am Aufkommen der ertragsstarken Steuern beteiligt wurden. Länder und Gemeinden erhielten zwei Drittel der Einkommenund Körperschaftsteuer, $20 \%$ der Erbschaftsteuer, $50 \%$

13 Bundesministerium der Finanzen: 100 Jahre Abgabenordnung, in: Monatsbericht des Bundesministeriums der Finanzen (BMF), November 2019, S. $43 \mathrm{f}$.

14 P.-C. Witt: Reichsfinanzminister und Reichsfinanzverwaltung. Zum Problem des Verhältnisses von politischer Führung und bürokratischer Herrschaft in den Anfangsjahren der Weimarer Republik (1918/19-1924), in: Vierteljahrshefte für Zeitgeschichte, 23. Jg. (1975), H. 1, S. $48 \mathrm{f}$.

15 R. van der Borght: Reichssteuergesetze 1919/20, S. 157 ff. der neu geschaffenen Grunderwerbsteuer und $15 \%$ der Umsatzsteuer. Dieses Verbundsystem besteht mit den "Gemeinschaftsteuern" bis heute - bei Einkommen- und Körperschaftsteuer, Umsatzsteuer sowie indirekt bei der Gewerbesteuer mit einer Umlage an Bund und Länder.

\section{Modernisierung des Steuersystems und massive Steuererhöhungen}

Die Lage der öffentlichen Finanzen war Mitte 1919 äußerst prekär. Das Reichsfinanzministerium schätzte den künftigen jährlichen Finanzbedarf auf 24 Mrd. Mark, davon 17,5 Mrd. für das Reich. ${ }^{16}$ Vor 1914 hatten die jährlichen Steuereinnahmen aller Gebietskörperschaften nur bei $4 \mathrm{Mrd}$. Mark gelegen. Durch die Steuererhöhungen im Krieg und die Inflation waren sie bis 1919 auf schätzungsweise 8 Mrd. Mark gewachsen. Die hohen laufenden Defizite von bis zu zwei Dritteln der Ausgaben wurden zunächst weiter über die Geld- und Kreditschöpfung mobilisiert. Das heizte die Inflation kräftig an und drohte die öffentlichen Finanzen zu zerrütten.

Erzberger machte die dramatische Finanzlage bei jeder Gelegenheit deutlich und verlangte massive Steuererhöhungen, um den Staatsbankrott abzuwenden. In 14 Gesetzen und weiteren Verordnungen wurden bis Ende März 1920 alle wesentlichen Steuern zentralisiert, modernisiert und deren Belastungen deutlich erhöht. Der Schwerpunkt sollte bei den „direkten“ Steuern auf Einkommen und Vermögen liegen, die mittelfristig drei Viertel des Steueraufkommens erzielen sollten. Die reichseinheitliche und progressive Einkommensteuer sollte die Haupteinnahmequelle werden, Vermögensteuern sollten die Wohlhabenden ergänzend belasten. Hier hatte es im Kaiserreich nur niedrige Belastungen gegeben. „Gerechtigkeit im gesamten Steuerwesen zu schaffen ist mein oberstes Ziel. (...) Ein guter Finanzminister ist der beste Sozialisierungsminister"17, erklärte Erzberger in seiner ersten Rede vor der Nationalversammlung.

Die Einkommensteuer wurde auf das Reich übertragen und massiv ausgebaut. ${ }^{18}$ Sie sollte gut ein Drittel der ausgeweiteten Steuereinnahmen erbringen und wurde nach langen Debatten zum Ende der Reformperiode im März 1920 verabschiedet. Eine historische Zäsur waren die breite Bemessungsgrundlage und der deutlich progressive Steuertarif, verglichen mit den herkömmlichen Belastungen der Länder-Einkommensteuern. Höhere und vor allem hohe Einkommen sollten kräftig belastet wer-

16 R. van der Borght: Reichssteuergesetze 1919, S. $136 \mathrm{ff}$.

17 M. Erzberger: Einführungsrede, a.a. O., S. 5.

18 R. van der Borght: Reichssteuergesetze 1919/20, S. 92 ff.; M. Hacker, a.a.O., S. 122 ff.; A. Schmidt-Essen: Reichseinkommensteuer, a.a. O. 
den, um die Ziele beim Steueraufkommen zu garantieren. Das Einkommenskonzept war an die „Reinvermögenszugangstheorie" angelehnt und damit weitreichender als die heutige Einkommensteuer. Es umfasste auch das selbstgenutzte Wohneigentum, Veräußerungsgewinne und Lotteriegewinne. Abgezogen werden konnten Werbungkosten, darunter auch Fahrtkosten zum Arbeitsort und Beiträge für Berufsverbände, sowie Vorsorgeaufwendungen, Spenden und Beiträge. Ferner wurden außergewöhnliche Belastungen anerkannt. Eingeführt wurde eine Haushaltsbesteuerung, bei der die Einkommen der Ehepartner zusammen veranlagt wurden, einschließlich der zum Haushalt zählenden Kinder, soweit sie keine Arbeitseinkommen bezogen.

Als Grundfreibetrag steuerfrei blieben 1500 Mark für eine einzelne Person sowie 500 Mark für jede weitere Person, die bei der Zusammenveranlagung einbezogen wurde, bzw. 700 Mark für Personen unter 16 Jahren. Für übersteigende Einkommen galt ein Stufentarif, der mit $10 \%$ einsetzte, über 50 weitere Stufen für die jeweils übersteigenden Einkommensintervalle um jeweils einen Prozentpunkt stieg und den Spitzensteuersatz von $60 \%$ ab steuerpflichtigen Einkommen von 500000 Mark erreichte. Das Durchschnittseinkommen rentenversicherungspflichtiger Arbeitnehmer lag 1919 bei 2000 Mark im Jahr. Anders als bei den bisherigen Einkommensteuergesetzen der Länder war damit die Steuerpflicht nicht nur auf die wohlhabenden Bürger beschränkt, sondern reichte bis weit in die Mittelschichten und zu gutverdienenden Facharbeitern herunter. Angesichts der bisher weit verbreiteten Untererfassung der Einkünfte sollte der Steuervollzug durch Quellensteuern wie die Lohnsteuer und die Kapitalertragsteuern effektiver gemacht werden. Die Besteuerung der juristischen Personen wurde in die Körperschaftsteuer ausgelagert. Der Körperschaftsteuersatz betrug $10 \%$, für ausgeschüttete Gewinne erhöhte sich der Steuersatz bis auf $20 \%$, die Doppelbelastung mit der Einkommensteuer wurde nicht gemildert.

Im Steuerausschuss und in der zweiten und dritten Lesung des Gesetzes stand dann vor allem der anvisierte direkte Abzug der Lohnsteuer vom Lohn im Mittelpunkt. Insbesondere die Deutschnationale Volkspartei (DNVP) und die Deutsche Volkspartei (DVP) lehnten das Vorhaben ab, weil sie dadurch einen zu großen Verwaltungsaufwand auf die Unternehmen zukommen sahen. Auch einige Angehörige der Regierungsparteien waren skeptisch, weil sie in der vorgezogenen Zahlungspflicht der Lohnabhängigen eine deutliche Benachteiligung erblickten. Für die Reform sprachen die erhebliche Verfahrensvereinfachung für die Verwaltung und die Vermeidung von Protesten, zu denen es immer wieder kam, wenn die Steuerbehörden zur Begleichung von Steuerrückständen ganze Wochen- löhne beschlagnahmten. Die Mehrheit der Regierungsfraktionen entschied sich deswegen für den direkten Lohnsteuerabzug, dessen Nachteile sie als nicht so gravierend ansahen, zumal ursprünglich nur ein Teil der abhängig Beschäftigten überhaupt steuerpflichtig gewesen wäre. Damit beschloss die Mitte-Links-Koalition letztlich jene Regelung, die bis zum Ende der Inflationsjahre zur stärksten Belastung für die abhängig Beschäftigten werden sollte. Denn die Inflation trieb in der Folgezeit fast alle Normalverdiener über das steuerfreie Existenzminimum. Dadurch, dass sie die Lohnsteuer direkt entrichteten, hatten sie erhebliche Nachteile gegenüber den Einkommensteuerveranlagten, deren Steuerbeträge auf das Einkommen des Vorjahres bei Erreichen der Zahlungspflicht inflationsbedingt erheblich an Wert eingebüßt hatten.

\section{„Reichsnotopfer" und Erbschaftsteuer}

Besonders kontrovers war das „Reichsnotopfer“, eine allgemeine Vermögensabgabe, mit der die exzessive Staatsverschuldung abgebaut werden sollte..$^{19}$ Sämtliche Vermögen abzüglich Schulden sollten erfasst werden. Als Freibetrag waren 5000 Mark für jede Person im Haushalt vorgesehen. Das entsprach 1919 schätzungsweise dem 2,5-fachen des Durchschnittseinkommens und wären heute etwa 100000 Euro. Der Steuertarif stieg progressiv von $12 \%$ für die ersten 50000 Mark steuerpflichtigen Vermögens bis auf $65 \%$ für steuerpflichtige Vermögen über 2 Mio. Mark. Juristische Personen wie Kapitalgesellschaften und Stiftungen zahlten $10 \%$. Die Abgabe sollte über 30 Jahre gezahlt werden, wobei die Ratenzahlungen mit $5 \%$ verzinst wurden, Abgabenbelastungen auf Grundbesitz konnten über 50 Jahre verteilt werden. Das Aufkommen wurde auf 70 Mrd. bis 90 Mrd. Mark geschätzt und sollte zumindest die inflationstreibende "schwebende“ Verschuldung im Bankensystem beseitigen.

Auch die Reform der Erbschaftsteuer war durch den hohen fiskalischen Bedarf motiviert, der vor allem bei den wohlhabenden Schichten realisiert werden sollte. ${ }^{20}$ Die Erbschaftsteuer war bereits ab 1906 durch das Reich vereinheitlicht worden, die Länder hatten aber Ertragsanteile und weitere Gestaltungsmöglichkeiten. Die Reform von 1919 übertrug die Steuer auf das Reich und machte erstmals auch Ehegatten und Kinder steuerpflichtig. Für sie galten eine Freigrenze von 5000 Mark und progressive Steuersätze von $4 \%$ bis $35 \%$. Dies hatten Junker und Großagrarier im Kaiserreich am schärfsten und erfolgreich bekämpft, weil sie die langfristige Sicherung des

19 A. Schmidt-Essen: Die große Einmalige, in: Wirtschaftsdienst, H. 30 vom 25.7.1919; R. van der Borght: Reichseinkommensteuergesetze 1919/20, a. a. O., S. $134 \mathrm{ff}$.

20 R. van der Borght: Reichseinkommensteuergesetze 1919, a.a.O., S. $192 \mathrm{ff}$. 
Familienbesitzes bedroht sahen. Bei ferneren Verwandten und Dritten galt nur eine Freigrenze von 500 Mark, der Spitzensteuersatz stieg auf $70 \%$ und wurde noch angehoben, wenn der Erbe Vermögen über 100000 Mark besaß. Zusätzlich wurde generell eine Nachlasssteuer auf Nachlässe über 20000 Mark erhoben, deren Steuersätze bei höheren Übertragungen bis auf $5 \%$ stiegen.

Ferner wurden die „indirekten“ Verbrauch- und Umsatzsteuern ausgeweitet, um diese Steuerquellen ebenfalls breit auszuschöpfen. ${ }^{21}$ Dies war vor allem bei den Sozialdemokraten unpopulär und brachte große Härten mit sich, angesichts grassierender Armut in großen Teilen der Bevölkerung durch die anhaltend schlechte Versorgungslage, die starken Preissteigerungen sowie die steigende Arbeitslosigkeit im Zuge der Demobilisierung. Die Umsatzsteuer wurde als allgemeine Verbrauchsteuer ausgebaut. ${ }^{22}$ Hierfür plante das Finanzministerium zunächst mit einem allgemeinen Steuersatz von $1 \%$ (bisher 0,5\%) sowie einem zusätzlichen Steuersatz von $5 \%$ auf den Endverbrauch. Dies wurde aber nicht umgesetzt, stattdessen der allgemeine Steuersatz auf 1,5\% und später schrittweise auf 2,5\% erhöht. Zusätzlich wurde der „Luxussteuersatz" für einzelne Produkte des gehobenen Bedarfs von $10 \%$ auf $15 \%$ angehoben. Ferner wurde die Grunderwerbsteuer eingeführt. Auch die speziellen Verbrauchsteuern wurden zum Teil empfindlich erhöht, vor allem die Tabaksteuer, die Zündwarensteuer, die Spielkartensteuer, die Schaumweinsteuer und die Post-, Telegraphen- und Fernsprechgebühren. Ergänzt wurde die Reform durch Gesetze gegen die Steuer- und Kapitalflucht. Das „Gesetz über Steuernachsicht" sah eine Amnestieregelung für frühere Steuerhinterziehung vor, wenn die Besteuerungsgrundlagen nachgemeldet wurden.

\section{Durchbruch zum modernen Steuer- und Wohlfahrtsstaat in Deutschland}

Die Steuer- und Finanzreformen waren zunächst fiskalisch nur wenig erfolgreich und konnten das Abrutschen in die Hyperinflation von 1923 nicht verhindern. Die neu entstehende Finanzverwaltung war am Anfang kaum in der Lage, die stark ausgeweiteten Steuern auf hohe Einkommen und Vermögen sachgerecht zu veranlagen. Viele der schnell entwickelten steuertechnischen Regelungen erwiesen sich als wenig praktikabel oder verbesserungsbedürftig. Bei den Steuerpflichtigen waren Bereitschaft und Möglichkeiten zur Mitwirkung gering. Die auch aus heutiger Sicht massiven Belastungen hoher Einkommen und Vermögen

21 Ebenda, S. 150 ff.; R. van der Borght: Reichseinkommensteuergesetze 1919/20, a.a.O., S. $80 \mathrm{ff}$

22 A. Schmidt-Essen: Die neue Umsatzsteuer, in: Wirtschaftsdienst, H. 34 vom 22.8.1919. durch Einkommensteuer, Reichsnotopfer oder Erbschaftsteuer schockierten das „Besitzbürgertum“ und die alten Eliten, insbesondere im Vergleich zu den bis dahin üblichen geringen Belastungen der Vorkriegszeit. Dies führte zu einer dramatischen Zunahme der Kapitalflucht, die in ähnlicher Weise auch in anderen Hochsteuerländern wie Großbritannien und Frankreich zu beobachten war. Dadurch etablierten sich die ersten Steueroasen dauerhaft, wobei die Schweiz die prominenteste Rolle einnahm. Für die deutsche Wirtschaftselite hatten zudem die Niederlande eine wichtige Funktion als Kapitalfluchthafen. ${ }^{23}$ Bis weit in die Mittelschichten hinein wurden nun viele Bürger mit Lohnsteuer und Einkommensteuer konfrontiert, die Sozialbeiträge stiegen und die ebenfalls kräftigen Erhöhungen von Umsatzsteuer und Verbrauchsteuern belasteten den Massenkonsum. Dies löste politischen Unmut und starken Steuerwiderstand aus. Zudem waren die Reparationen verhasst, die man nicht mit hohen Steuern mitfinanzieren wollte. Bei vielen konservativen Zeitgenossen galt das für die Republik generell.

Vor allem entwertete die weiter steigende Inflation die Steuerzahlungen. Daher scheiterten das Reichsnotopfer und die Erhöhungen bei der Erbschaftsteuer in den Folgejahren weitgehend. Dies galt teilweise auch für die neue Einkommensteuer. Ab 1923 wurde das Reichsnotopfer durch die Vermögensteuer abgelöst, die bis 1996 erhoben wurde. Die Erbschaftsteuer wurde in den Folgejahren deutlich entlastet. Man halbierte 1922 den Höchstsatz der Erbanfallsteuer bei den nächsten Verwandten, was vor allem erbenden Kindern zugutekam, denn die Ehegatten wurden mit der Reform bis auf wenige Ausnahmen wieder vollständig steuerfrei gestellt. ${ }^{24}$ So spielte die Erbschaftsteuer im Gegensatz zu Großbritannien und den USA für das Steueraufkommen weiterhin nur eine untergeordnete Rolle und ermöglichte so die weitgehend unbelastete Weitergabe großer Vermögen.

Erzberger selbst erlebte die Weiterentwicklung seiner Reformen nicht mehr. Nach persönlichen Angriffen durch den rechtskonservativen früheren Staatssekretär des Reichsschatzamtes, Karl Helfferich, der vor Gericht wegen seiner Beleidigungen nur zu einer geringen Geldstrafe verurteilt wurde, trat er im März 1920 zurück. Erzberger wurde zur Symbolfigur der in bürgerlich-konservativen Kreisen zumeist abgelehnten Republik. Dazu trugen wohl auch die Steuer- und Finanzreformen maßgeblich bei. Zudem wurde Erzberger von der rechten Propaganda als „Novemberverbrecher" geschmäht, weil er sich in den

23 C. Farquet: La Défense du paradis fiscal suisse avant la seconde guerre mondiale: une histoire internationale, Neuchatel 2016.

24 J. Beckert: Unverdientes Vermögen. Soziologie des Erbrechts, Frankfurt a. M. 2004, S. 273 f. 
letzten Kriegsjahren für einen Verständigungsfrieden eingesetzt und im November 1918 die deutsche Delegation geleitet hatte, die den Waffenstillstand unterzeichnete. Im August 1921 wurde er von Rechtsradikalen ermordet, die ins Ausland fliehen konnten und 1933 von den Nationalsozialisten amnestiert wurden.

Nach der Währungsstabilisierung 1924 sicherten die Steuer- und Finanzreformen dann aber die Konsolidierung der öffentlichen Finanzen. Diese gelang durch die inzwischen verbesserte Steuererhebung und konsequente Ausgabendisziplin. Im Zuge weiterer Reformen wurden in den nächsten Jahren zahlreiche steuertechnische Mängel und Übergangsregelungen beseitigt. ${ }^{25}$ Ab 1925 reduzierte die regierende bürgerlich-konservative Koalition die Steuerbelastungen, der Spitzensatz der Einkommensteuer sank auf $40 \%$, der Umsatzsteuersatz auf 0,75\%. Einkommensteuer und Körperschaftsteuer wurden steuertechnisch überarbeitet, das Bewertungsgesetz schaffte einheitliche Besteuerungsgrundlagen für sämtliche vermögensbezogenen Steuern.

Im Jahr 1925 lag die gesamtwirtschaftliche Steuerbelastung bei $15 \%$ des BIP und hatte sich damit gegenüber den Jahren vor dem Ersten Weltkrieg nahezu verdoppelt. ${ }^{26}$ Auch die Sozialbeiträge waren gestiegen und wurden weiter erhöht, vor allem durch die Einführung der Arbeitslosenversicherung 1927. In den Grundstrukturen entstand damit nach den Erzbergerschen Reformen das heutige Steuersystem mit Umsatzsteuer und speziellen Verbrauchsteuern einerseits sowie den Einkommensteuern einschließlich Körperschaft- und Gewerbesteuer andererseits als den beiden Grundpfeilern des Steuerstaats. Hinzu kamen die vermögensbezogenen Steuern, die damals ein viel größeres Gewicht hatten als heute. Dies betraf Grunderwerbsteuer, Kapitalverkehrssteuern, Grundsteuer, die Vermögensteuer, Erbschaftsteuer und die Ge-

25 M. Buggeln: Die Debatten um staatliche Zugriffsmöglichkeiten auf den privaten Reichtum. Der Einkommensteuerspitzensatz in Deutschland 1871-1955, in: Vierteljahrschrift für Sozial- und Wirtschaftsgeschichte, 105. Jg. (2018), H. 3, S. 337-364; M. Hacker, a.a. O., S. 201 ff.; F. Terhalle: Zur Reichsfinanzreform von 1925, in: Zeitschrift für die gesamte Staatswissenschaft, 80. Jg. (1925), S. 289-340.

26 S. Bach: 100 Jahre deutsches Steuersystem. Revolution und Evolution, DIW Discussion Paper, Nr. 1767, Berlin 2018, S. 6; ders.: 100 Jahre deutsches Steuersystem: Grundlagen, Reformen und Herausforderungen, in: DIW Wochenbericht, Nr. 47/2019, S. 858-863. bäudeentschuldungssteuern der Länder (Hauszinssteuer), die Schuldnergewinne der Immobilienbesitzer nach der Währungsumstellung abschöpften.

Die Wirtschafts- und Sozialpolitik der Weimarer Republik war im Vergleich zum Kaiserreich deutlich interventionistischer und wohlfahrtsstaatlicher ausgerichtet. Die sozialen Sicherungssysteme und die öffentliche Daseinsvorsorge wurden ausgebaut. In diesem Kontext entstand eine intensive Diskussion über die Tragfähigkeit der hohen Steuerund Abgabenbelastungen für Wirtschaft und Bevölkerung, die den heutigen Debatten gleicht. ${ }^{27}$ Wirtschaftsverbände sowie liberale und konservative Politiker verlangten Entlastungen, während Sozialkonservative, Linksliberale, Sozialdemokraten und Gewerkschaften die wohlfahrtsstaatlichen Errungenschaften erhalten und ausbauen wollten.

\section{Revolution und Evolution des deutschen Steuersystems}

Vor 100 Jahren zentralisierten und modernisierten die „Erzbergerschen Steuer- und Finanzreformen“ das Steuersystem und die Finanzverfassung in Deutschland umfassend und schufen damit Grundlagen, die bis heute wirksam sind. Die Belastungen mit Steuern und Sozialbeiträgen verdoppelten sich. Seit dieser Revolution verliefen die Steuerreformprozesse evolutionär. NS-Regime und Zweiter Weltkrieg erhöhten die Belastungen weiter. Seit den 1950er Jahren bewegte sich die gesamtwirtschaftliche Steuerbelastung in Deutschland meist zwischen $22 \%$ und $24 \%$ des BIP. Auch die Grundstrukturen des Steuersystems und der Finanzverfassung blieben seitdem weitgehend erhalten.

Die einzelnen Steuern und die Zusammensetzung der Steuerbelastungen wurden allerdings häufig verändert. Größere Steuerreformen waren der Übergang bei der Umsatzsteuer zur Mehrwertsteuer Ende der 1960er Jahre, die Einkommen- und Körperschaftsteuerreformen der 1970er Jahre, die schrittweisen Einkommensteuerreformen von 1996 bis 2005, die Unternehmensteuerreformen 2001 und 2008, die Erhöhung der Mehrwertsteuer in den 1990er und 2000er Jahren sowie die Ausweitung der Energiesteuern 1999 bis 2003.

27 H.-P. Ullmann, a.a. O., S. $123 \mathrm{ff}$

Title: Birth of the Modern Tax State in Germany 1919-1920

Abstract: 100 years ago, the "Erzberger fiscal reforms" fundamentally reshaped public finances in Germany. They centralised the fiscal constitution and modernised the tax system comprehensively. Personal income tax corporate income tax, and inheritance tax were harmonised nationwide and significantly increased. Moreover, a capital levy was introduced and indirect taxes were increased. The overall tax burden has doubled since 1925. These reforms shape the foundations of public finances and the tax system to this day.

JEL Classification: N44, H2O, H11 